\title{
In depth nano spectroscopic analysis on homogeneously switching double barrier
}

memristive devices

Julian Strobel $^{\mathrm{a}}$, Mirko Hansen ${ }^{\mathrm{b}}$, Sven Dirkmann $^{\mathrm{c}}$, Krishna Kanth Neelisetty ${ }^{\mathrm{d}}$, Martin Ziegler ${ }^{\mathrm{b}}$, Georg Haberfehlner ${ }^{\mathrm{e}}$, Radian Popescu ${ }^{\mathrm{f}}$, Gerald Kothleitner $^{\mathrm{e}}$, Venkata Sai Kiran Chakravadhanula $^{\mathrm{d}}$, Christian Kübel ${ }^{\mathrm{d}}$, Hermann Kohlstedt ${ }^{\mathrm{b}}$, Thomas Mussenbrock ${ }^{\mathrm{g}}$, and Lorenz Kienle ${ }^{\mathrm{a}, *}$

a: Synthesis and Real Structure, University of Kiel, Faculty of Engineering, Kaiserstr. 2, 24143 Kiel, Germany

just@tf.uni-kiel.de;, lk@tf.uni-kiel.de

b: Nanoelectronics, University of Kiel, Faculty of Engineering, Kaiserstr. 2, 24143 Kiel, Germany mha@tf.uni-kiel.de,maz@tf.uni-kiel.de,hko@tf.uni-kiel.de c: Ruhr Universität Bochum, Faculty for Electrical Engineering and Information Technology, Universitätsstr. 150, 44801 Bochum, Germany sven.dirkmann@gmx.de, mussenbrock@gmail.com

d: Karlsruhe Institute of Technology, Institute of Nanotechnology, Hermann-von-Helmholtz-Platz 1, 76344 Eggenstein-Leopoldshafen, Germany cvskiran@kit.edu, krishna.neelisetty@kit.edu e: Technical University Graz, Institute for Electron Microscopy and Nanoanalytics, Steyrergasse 17, 8010 Graz, Austria georg.haberfehlner@felmi-zfe.at, gerald.kothleitner@felmi-zfe.at f: Karlsruhe Institute of Technology, Laboratory of Electron Microscopy Engesserstr. 7, 76131 Karlsruhe, Germany popescu@kit.edu g: Brandenburg University of Technology, Electrodynamics and Physical Electronics 03046 Cottbus, Germany 
mussenbrock@b-tu.de

*Corresponding author:

Lorenz Kienle, Kaiserstr. 2, 24143 Kiel, Germany

Email: 1k@tf.uni-kiel.de; Phone: 00494318806196

\begin{abstract}
Memristors based on a double barrier design have been analysed by various nano spectroscopic methods to unveil details about its microstructure and conduction mechanism. The device consists of an $\mathrm{AlO}_{\mathrm{x}}$ tunnel barrier and a $\mathrm{NbO}_{\mathrm{y}} / \mathrm{Au}$ Schottky barrier sandwiched between $\mathrm{Nb}$ bottom electrode and $\mathrm{Au}$ top electrode. As it was anticipated that the local chemical composition of the tunnel barrier, i.e. oxidation state of the metals as well as concentration and distribution of oxygen ions, have a major influence on electronic conduction, these factors were carefully analysed. A combined approach was chosen in order to reliably investigate electronic states of $\mathrm{Nb}$ and $\mathrm{O}$ by electron energy-loss spectroscopy as well as map elements whose transition edges exhibit a different energy range by energydispersive X-ray spectroscopy like $\mathrm{Au}$ and $\mathrm{Al}$. The results conclusively demonstrate significant oxidation of the bottom electrode as well as a small oxygen vacancy concentration in the $\mathrm{Al}$ oxide tunnel barrier. Possible scenarios to explain this unexpected additional oxide layer are discussed and kinetic Monte Carlo simulations were applied in order to identify its influence on conduction mechanisms in the device. In light of the strong deviations between observed and originally sought layout, this study highlights the robustness in terms of structural deviations of the double barrier memristor device.
\end{abstract}

Keywords: memristor, TEM, EELS, EDX 


\section{INTRODUCTION}

The scientific interest in resistive switching devices increases drastically when in 2008 Strukov et al. claimed that the fourth lumped circuit element has been found. [1] It was theoretically predicted by Chua in the early 1970s. [2] This device also referred to as the memristor has opened the door to a vast number of new and promising applications. The applications of memristors span from computer memories (RRAM) over computational pattern recognition to neuromorphic engineering. [3-6]

Unlike the transistor which initiated the electronic revolution and which of course has seen manifold modifications since its introduction, there is no single common design-scheme for memristive devices. Furthermore, the physical mechanisms which are responsible for the drastic change of the resistance are diverse as well. A categorization can be made based on the resistive switching mechanism: in electrochemical metallization (ECM) cells a conductive filament forms from what is coined the active electrode towards the inactive electrode. The active electrode and its conductive filament consists of non-inert metals like $\mathrm{Ag}$ or $\mathrm{Cu}$. Inactive electrodes in all these devices may consist of noble metals such as $\mathrm{Au}$ and $\mathrm{Pt}$ or from conductive compounds, e.g. TiN. [7-10] In valence change memory (VCM) cells transition metal oxides between the electrodes locally change their oxidation state by movement of charged defects, such as oxygen vacancies. This behaviour can occur in the form of homogeneous drift of charge carriers or filamentary switching. [11,12] Phase change memory (PCM) devices exhibit the formation of crystalline filaments in an amorphous matrix. This behavior can be observed in chalcogenides such as complex tellurides [13] and e.g. in amorphous TiOx where crystalline Magnéli filaments form. [14] Of course this categorization is not complete as other less common types of switching exist, and like any classification it is far from absolute; e.g. a phase change can - and often does - include a valence change. Among these approaches two-terminal tunnel junctions [15-17] represent a very promising 
approach as they might be directly compatible to CMOS technology and work with small voltages and currents, hence consume only little power.

An elegant realization of a memristive device which does not rely on the disadvantageous formation and deformation of filaments has been proposed by Hansen et al. [16] It consists of a double tunnel barrier with a variable tunnel resistance. (cf. Figure 1) The conduction mechanism and switching principle are matter of ongoing research. Electrical characterization and numerical simulations [18] which has been already performed prove the concept. However, a profound experimental investigation from the nanoanalytical point of view has not been reported yet; consequently, the memristive double barrier device is investigated based on a dedicated electron microscopic analysis. A particular experimental challenge is to investigate nanoscale chemical segregation phenomena in the very thin amorphous layers which exhibit thicknesses around $10 \mathrm{~nm}$. The determination of such features is highly relevant, since it is expected that mobile oxygen ions in the Schottky and the tunnel barrier are responsible for the resistance change. In order to address these open questions the experimental results have been analyzed in conjunction with Monte Carlo simulations of the device.

\section{EXPERIMENTAL}

\subsection{Device fabrication}

A stack of $\mathrm{Nb} / \mathrm{Al}-\mathrm{AlO}_{\mathrm{x}} / \mathrm{NbO}_{\mathrm{y}} / \mathrm{Au}$ was deposited onto thermally oxidized $\mathrm{Si}-\mathrm{SiO}_{2}$ wafers. The multilayer devices were fabricated by standard optical lithographic means and DC magnetron sputtering onto 4-inch wafers. Detailed deposition parameters can be found in [16]. The targeted thicknesses were $6 \mathrm{~nm}(\mathrm{Al}), 1 \mathrm{~nm}\left(\mathrm{AlO}_{\mathrm{x}}\right), 1.2 \mathrm{~nm}\left(\mathrm{NbO}_{\mathrm{y}}\right) . \mathrm{Nb}$ and $\mathrm{Au}$ serve as bottom and top electrode and their thicknesses are large compared to the $\mathrm{Al}$ and oxide films. A schematic of the targeted layout is depicted in Figure 1 (b). Because the design of the 
memristive device is identical to superconducting Josephson junctions except for the lack of the second oxide layer (cf. Figure 1 (c)), it has been analyzed in this study to the effect of validating results, because it is a well-understood system under analysis for decades.

\subsection{TEM sample preparation}

Cross-sectional samples were prepared by focused ion beam (FIB) milling on a FEI Helios NanoLab as well as FEI Strata $400 \mathrm{~S}$ and thinned down to thicknesses of about 100-150 nm. Final thinning was done by treatment with low energy Ar ions in a Fischione NanoMill to a final thickness of around $50-80 \mathrm{~nm}$ to prohibit any preparation artifacts from the FIB's Ga ions. Josephson junction wafers were also available as large scale samples for which crosssectional sample preparation by Precision Ion Polishing System (PIPS) was applicable. As PIPS is expected to be less prone to preparation induced artifacts, a comparative study between FIB and PIPS prepared samples was conducted. In order to substantiate results even further, FIB backside thinning was applied to exclude any directional thinning artifacts, e.g. oxidation of the metal layers.

\subsection{TEM investigations and data analysis}

As the overall thickness of the critical layers in the double barrier memristor (DBM) is well below $10 \mathrm{~nm}$ and information both on the structure and local chemistry is required transmission electron microscopy (TEM) analysis was conducted. The chemical information was retrieved by energy-dispersive X-ray spectroscopy (EDX) and electron energy-loss spectroscopy (EELS) in scanning TEM (STEM). High-angle annular dark field (HAADF)STEM combined with EDX experiments were performed on a FEI Osiris ChemiSTEM microscope at $200 \mathrm{kV}$ acceleration voltage, which is equipped with a Bruker Quantax system (XFlash detector) for EDX. Elemental maps of $\mathrm{Al}\left(\mathrm{Al}-\mathrm{K}_{\alpha}\right.$ line $), \mathrm{Nb}\left(\mathrm{Nb}-\mathrm{K}_{\alpha}\right)$ and $\mathrm{O}\left(\mathrm{O}-\mathrm{K}_{\alpha}\right.$ line) were recorded and used to investigate their distribution within multi-layers. The maps were analyzed by using the ESPRIT software (version 1.9) from Bruker. Using ESPRIT, 
element concentrations were calculated on the basis of a refined Kramers' law model, which includes corrections for detector absorption and background subtraction. For this purpose, standard-less quantification, i.e. by means of theoretical sensitivity factors, without thickness correction was applied.

Combined STEM-EELS and EDX experiments were carried out on a FEI Titan ${ }^{3}$ (monochromator, XFEG, GIF Quantum energy analyzer, SuperX EDX detector). The energy resolution during EELS was continuously checked to be at least $0.3 \mathrm{eV}$. Real space micrographs were taken by annular dark field imaging also in STEM mode. Data processing of the EEL spectra was undertaken with Gatan Microscopy Suite 3 and HyperSpy [19]. The data was treated by principal component analysis to reduce noise and find all significant features.[20] Oxygen K spectra were fitted with a multiple linear least square (MLLS) fit to a reference spectrum in order to locate specific oxides. The references were extracted from the middle of the respective oxide layers $(\mathrm{Nb}$ and $\mathrm{Al})$ and then fit to the rest of the EELS data creating a goodness of fit map.

\subsection{Anodization spectroscopy}

The anodization of thin films through the application of a constant direct current and the simultaneous measurement of the resulting voltage allows investigating layer properties (e.g. thickness and roughness). Anodization spectroscopy has been applied successfully to optimize the fabrication of high-quality low-temperature all-niobium Josephson tunnel junctions.[21] The set-up for anodization spectroscopy consists of an electrolyte bath, an Au electrode (cathode), the sample of interest (anode), a current source and a computer for data acquisition.[22] The most common method is to record the measured voltage V over time and subsequently plot the time derivative $\mathrm{dV} / \mathrm{dt}$ (anodization rate) versus $\mathrm{V}$ at room temperature. Different materials exhibit different anodization rates, leading to a characteristic anodization spectrum were the bulk materials and the interface between layers are easily distinguishable. 
The low-cost set-up together with the possibility to explore interfacial properties on the nanometer level and the lack of time consuming sample preparation (e.g. annealing, patterning etc.) made anodization spectroscopy an established and attractive method.[23] Although not all features of anodization profiles are understood yet, the different anodization rates of different materials and the transition between two layers allows comparative studies. Therefore, we applied anodization spectroscopy to study memristive double barrier $\mathrm{Nb} / \mathrm{Nb}_{\mathrm{x}} \mathrm{O}_{\mathrm{y}}$ $/ \mathrm{Al}_{2} \mathrm{O}_{3}-\mathrm{Al} / \mathrm{Nb}$ junctions. The top electrode had to be replaced by $\mathrm{Nb}$ because of the noble character of Au making it unsuitable for anodization spectroscopy.

\section{RESULTS}

\subsection{Structural and spectroscopic analysis of DBM devices}

After ensuring that the preparation methods are generally suitable for this material system the analysis of the actual memristive device was conducted. A high resolution micrograph revealing the metallic crystalline and amorphous oxide regions is depicted in Figure 2 (d). The indicated thickness of $10 \mathrm{~nm}$ of the amorphous area (dashed lines) containing three distinct oxides is remarkable as it is much thicker than expected and unusually thick for a functioning tunnel barrier. Subsequently, chemical information of the sample was extracted by STEM-EDX experiments. Here, it is clearly visible (cf. Figure 2 (a) and (b)) that the $\mathrm{Nb}$ bottom electrode exhibits oxidation reaching several nanometers in thickness (labelled $\mathrm{NbO}_{\mathrm{z}}$ in the following). Furthermore, no isolated metallic $\mathrm{Al}$ seems to be present as apparent from the lateral distribution of oxygen. The $\mathrm{NbO}_{\mathrm{y}}$ deposited on top of the $\mathrm{AlO}_{\mathrm{x}}$, is ca. $1 \mathrm{~nm}$ thick and thus closely corresponds to the thickness predicted from deposition parameters; the combined thickness of the three oxide layers is about $10 \mathrm{~nm}$ and corresponds with the amorphous region visible in the HRTEM micrograph. The surface roughness clearly visible between the lower $\mathrm{NbO}_{z}$ and $\mathrm{AlO}_{\mathrm{x}}$ is also in line with expectations and is similar to that observed for the Josephson junction (cf. supplement). A slight overlap of the $\mathrm{NbO}_{\mathrm{z}}$ and $\mathrm{AlO}_{\mathrm{x}}$ 
is present and may be caused by two factors. Firstly, the surface roughness causes a superposition of $\mathrm{Nb}$ and $\mathrm{Al}$ layers along the beam direction thus signal mixing for $\mathrm{NbO}_{\mathrm{z}}$ and $\mathrm{AlO}_{\mathrm{x}}$ is produced from the same apparent lateral position. Secondly, a slight interdiffusion may be possible. However, taking into account the tiny dimensions of the layers it seems hardly possible to differentiate between these two cases.

The source of $\mathrm{Nb}$ bottom electrode (BE) oxidation is unknown. Samples have been prepared by FIB milling both from the front- as well as from the backside, with and without NanoMill treatment in order to test for a Ga-beam induced oxidation during FIB preparation. As samples from all preparation routes yielded qualitatively the same results in the TEM, a directional - i.e. beam induced - artefact can be excluded (see supplementary material). Furthermore, the congruency of results between FIB and PIPS prepared Josephson junction suggests that the FIB does not alter the thin $\mathrm{NbO}_{\mathrm{y}}$ or $\mathrm{AlO}_{\mathrm{x}}$ layers; PIPS preparation is expected to cause no artifacts due to the use of low energy Ar ions.

Additionally, STEM-EELS investigations were conducted using a narrow energy range containing the O-K edge $(532 \mathrm{eV})$, hence, more detailed chemical information was available. The $\mathrm{O}-\mathrm{K}$ edge was fitted to reference spectra from $\mathrm{Al}$ oxide and $\mathrm{Nb}$ oxide. The references were extracted according to the method described in section 2.3. Maps of the goodness of this fit are depicted in Figure 3, clearly identifying the locations where oxygen is present in the form of $\mathrm{AlO}_{\mathrm{x}}$ and $\mathrm{NbO}_{\mathrm{y} / \mathrm{z}}$, conclusively proving the oxidation of the $\mathrm{BE}$ as well as the absence of metallic Al. Most importantly though, the fits allow determination of the true thickness of the tunnel barrier at its thinnest locations. Due to 3D surface roughness, the thinnest areas of the tunnel barrier are always obscured by thicker regions along the beam direction. However, the extent of $\mathrm{Nb}$ oxide as determined from the $\mathrm{O}-\mathrm{K}$ edge, proves that the tunnel barrier is in fact only around $1 \mathrm{~nm}$ thick at the thinnest spots, allowing tunnelling as proposed by Hansen et al. The yellow lines in Figure 3 (a) indicate these regions. As the $\mathrm{NbO}_{\mathrm{y}}$ Schottky barrier exhibits no significiant surface roughness, the maximum surface roughness extent of the 
$\mathrm{NbO}_{\mathrm{z}}$ (at the location below the yellow lines) implies the minimum thickness of the tunnel barrier.

Aside from the lateral distribution of elements the near edge structure (ELNES) of the O-K edge was analyzed in more detail. The reference spectra, also used for MLLS fitting of the maps shown in Figure 3, are displayed in Figure S1. The $\mathrm{AlO}_{\mathrm{x}}$ spectra all generally resemble the $\mathrm{Al}_{2} \mathrm{O}_{3}$ spectrum known from literature, which is no surprise as $\mathrm{Al}$ oxide is basically always a stoichiometric oxide and no sub- or superoxide. However, a pre-peak feature of the O-K edge at $538.6 \mathrm{eV}$ emerges, shifted about $6 \mathrm{eV}$ to lower energies compared to the main edge. This feature appears to be characteristic since it was observed across all samples. A close up of this feature is shown in Figure 4 along with two fit lines for the main edge and its pre-peak. It has been reported in literature that irradiation by the electron beam can cause damage to $\mathrm{Al}$ containing oxides and produce similar features.[24,25] However, these spectra were recorded on a monochromated instrument and the dwell times were only $100 \mathrm{~ms}$, thus irradiation damage should be marginal. Furthermore, no timely evolution of this feature was observed, which is expected for beam induced processes. This makes it improbable for this feature to be induced during the investigation. The possibility of this pre-peak emerging due to the influence of either of the neighbouring $\mathrm{Nb}$ oxides [26] was also considered. However, no $\mathrm{Nb}-\mathrm{M}$ edge, neither M4/5, M3/2 nor M1, was observed, which were all perfectly visible in the $\mathrm{NbO}_{\mathrm{y} / \mathrm{z}}$ regions. Furthermore, the pre-peak feature is present throughout the entire $\mathrm{AlO}_{\mathrm{x}}$ layer and not only at the interface to neighbouring $\mathrm{Nb}$ containing layers, hence, this scenario is excluded for interpretation.

As Nigo et al. [27] described in their study, though, such a feature could be indicative of oxygen vacancies. The electronic structure of alumina [28] and the influence of oxygen vacancies [29-31] on it have been described extensively in the literature. Accordingly, the area ratio of pre-peak to main edge is characteristic for the concentration of oxygen vacancies. As described by Johnson and Pepper, mid-band gap states in $\mathrm{Al}_{2} \mathrm{O}_{3}$ belong to localized $\mathrm{Al}(3 \mathrm{~s})$ 
states, which usually form bonding $\mathrm{O}(2 \mathrm{p})-\mathrm{Al}(3 \mathrm{~s})$ states and are located in the valence band. [32] The lower boundary of the conduction band is formed by the $\mathrm{Al}(3 \mathrm{p})$ orbitals, accordingly this corresponds to the main peak of the $\mathrm{O}-\mathrm{K}$ edge. As $\mathrm{Al}(3 \mathrm{p})$ is threefold degenerate, three times the number of the $\mathrm{Al}(3 \mathrm{p})$ states compared to overall - i.e. bound $-\mathrm{Al}(3 \mathrm{~s})$ states exist. Under the approximation that oxygen 1s electrons are excited into $\mathrm{Al}(3 \mathrm{~s})$ and $\mathrm{Al}(3 \mathrm{p})$ states with equal probability, the integrated EELS intensities scale linearly with the number of states $N_{A l(o r b .)}$ described above. Accounting for the local symmetry of $\mathrm{Al}_{2} \mathrm{O}_{3}$ every oxygen ion is coordinated with four aluminum ions; one vacancy hence produces four localized $\mathrm{Al}(3 \mathrm{~s})$ states. The total concentration of oxygen vacancies $c_{v a c}$ is therefore given by:

$$
\frac{3}{4} \cdot \frac{N_{A l(3 s), l o c}}{N_{A l(3 p)}}=\frac{1}{4} \frac{N_{A l(3 s), l o c}}{N_{A l(3 s)}}=c_{v a c}
$$

Where the ratio of localized $\mathrm{Al}(3 \mathrm{~s})$ to $\mathrm{Al}(3 \mathrm{p})$ (highlighted in green) is the value experimentally determined from ELNES analysis (cf. Figure 4). Oxygen vacancy concentrations were calculated accordingly, yielding integrated EELS intensities $I_{A l(3 s), l o c} / I_{A l(3 p)}$ between $2.2 \%$ and $4.3 \%$ over different samples which results in oxygen vacancy concentrations of $1.7 \%$ to $3.2 \%$. Since literature on this kind of quantification is scarce and verification of this result with samples of known concentrations was never reported, the accuracy of these values can only be estimated. Especially the relative scattering probability into bonding $\mathrm{Al}(3 \mathrm{p})$ and localized $\mathrm{Al}(3 \mathrm{~s})$ orbitals is unknown. However, as the oxide is completely amorphous, differences from anisotropy of the electronic structure can excluded. Furthermore, both orbitals are completely empty, hence Coulomb repulsion or effects from electron spin can be excluded.

Identification of $\mathrm{Al}$ and $\mathrm{Nb}$ oxidation state was done by comparison with O-K ELNES spectra from literature [33,34]. As expected, the $\mathrm{O}-\mathrm{K}$ near edge structure in the $\mathrm{AlO}_{\mathrm{x}}$ region resembles that of $\mathrm{Al}_{2} \mathrm{O}_{3}$ and $\mathrm{NbO}_{\mathrm{z}}$ region resembles that of $\mathrm{Nb}(\mathrm{II})$ oxide the most. The $\mathrm{O}-\mathrm{K}$ ELNES from the $\mathrm{NbO}_{\mathrm{y}}$ barrier could not be identified as a specific $\mathrm{Nb}$ oxide, a mixture 
between $\mathrm{Nb}(\mathrm{IV})$ and $\mathrm{Nb}(\mathrm{V})$ seems likely, though. (cf. Figure 5) Comparison of edge cross sections supports the ELNES comparison, as the $\mathrm{O} / \mathrm{Nb}$ ratio is $25 \%$ higher in the $\mathrm{NbO}_{\mathrm{y}}$ Schottky barrier oxide than in the oxidized bottom electrode. Thus the degree of oxidation is higher in $\mathrm{NbO}_{\mathrm{y}}$ with an expected oxidation state to be at least $\mathrm{Nb}(\mathrm{IV})$. This, too, underlines the stability of the Schottky barrier at the $\mathrm{NbO}_{\mathrm{y}} / \mathrm{Au}$ interface.

\subsection{Anodization spectroscopy}

The black curve in Figure 6 shows a typical anodization spectrum of an $\mathrm{Nb} / \mathrm{Al}_{2} \mathrm{O}_{3}-\mathrm{Al} / \mathrm{Nb}$ Josephson junction.[35] The anodization front propagates from the surface of the upper $\mathrm{Nb}$ layer. When the anodization front reaches the $\mathrm{Al}_{2} \mathrm{O}_{3}$ tunnel barrier at around $10-11 \mathrm{~V}$, the anodization rate increases sharply (peak 1). The weak minimum observed at $13.5 \mathrm{~V}$ is attributed to the $\mathrm{Al}_{2} \mathrm{O}_{3} / \mathrm{Al}$ interface and a second maximum (peak 2) indicates an excess $\mathrm{Al}$ layer not oxidized during barrier formation. The smeared and weak minimum between $16 \mathrm{~V}$ and $18 \mathrm{~V}$ (see inset of Figure 6) is attributed to the $\mathrm{Al} / \mathrm{Nb}$ interface and reflects the wetting effect of sputtered $\mathrm{Al}$ on polycrystalline $\mathrm{Nb}$ films. [34, 35]

The anodization spectrum of the memristive device $\mathrm{Nb} / \mathrm{Nb}_{\mathrm{x}} \mathrm{O}_{\mathrm{y}} / \mathrm{Al}_{2} \mathrm{O}_{3}-\mathrm{Al} / \mathrm{Nb}$ (green curve) shows all the above-mentioned features, except for a significantly larger peak 1 . This can be explained by the high resistance of the oxygen-rich niobium oxide layer, because the voltage necessary to drive the current has to increase rapidly. This significant increase and decrease in anodization rate makes it difficult to investigate the quality of the underlying $\mathrm{Al}_{2} \mathrm{O}_{3}$ tunneling barrier. Given the broadened shape of peak 1 and the resolution of the anodization spectroscopy, it is difficult to estimate the influence of the reactive sputtering of $\mathrm{NbO}_{\mathrm{y}}$ on the $\mathrm{Al}_{2} \mathrm{O}_{3}$ tunnel barrier. Nevertheless, the DBM shows the same transition from the aluminum to the niobium layer as the Josephson junction (around $15-17 \mathrm{~V}$ ). This suggests that the aluminum layer is not completely oxidized. Furthermore, the lack of an additional $\mathrm{Nb}$ oxide peak suggests the metallic low-resistance character of the $\mathrm{Al}_{2} \mathrm{O}_{3}$-back electrode interface. 
To understand how an oxidized aluminum layer would influence the anodization spectrum, a further aluminum oxide layer was introduced: After one third of the regular Al thickness, the deposition was stopped and 1 mbar of oxygen was introduced (i.e. $1 \%$ of the pressure of the actual $\mathrm{Al}_{2} \mathrm{O}_{3}$ tunnel barrier). Afterwards, the remaining two-thirds of the aluminum layer was sputtered and oxidized as above, yielding the $\mathrm{Nb} / \mathrm{Al}_{2} \mathrm{O}_{3}-\mathrm{Al} / \mathrm{Al}_{2} \mathrm{O}_{3}-\mathrm{Al} / \mathrm{Nb}$ multilayer (red curve). The red curve in Figure 6 shows the significant change compared to the Josephson junction and the DBM. This indicates that even the $\mathrm{Al}$ oxidized at 1 mbar is observable using anodization spectroscopy.

Anodization spectroscopy allows qualitative analysis of layer oxidation. To investigate the influence of the reactive sputtering on the electrical properties of the bottom electrode, samples with the following layer sequences were fabricated: 1) $\mathrm{Al}_{2} \mathrm{O}_{3}-\mathrm{Al} / \mathrm{Nb}$ and 2) $\mathrm{Nb}_{\mathrm{x}} \mathrm{O}_{\mathrm{y}} / \mathrm{Al}_{2} \mathrm{O}_{3}-\mathrm{Al} / \mathrm{Nb}$. The thickness of the bottom $\mathrm{Nb}$ electrode was reduced to approximately $4 \mathrm{~nm}$, to emphasize the impact of the reactive sputtering. Afterwards, the resistance of both thin films was measured. If the reactive deposition is assumed to have a significant influence on the bottom electrode, this should lead to a drastic increase in film resistance, because the total multilayer thickness is comparable to the thickness of the oxidized layers observed in the STEM-EELS measurements. Both layers, however, showed ohmic behavior and had an almost identical resistance: Sample 1 had a resistance of $(636+/-50) \mathrm{k} \Omega$ while sample 2 had a resistance of $(635+/-43) \mathrm{k} \Omega$. This further confirms the metallic nature of the bottom electrode and is congruent to the observation of metallic character $\mathrm{Nb}$ suboxide in TEM investigations.

\subsection{Kinetic Monte Carlo simulations}

Within presented simulation approaches up to now, the resistances of the electrodes has been neglected. Models based on this device structure were able to explain important physical processes during the resistance change of the DBM device. [18] Although experimental results are not unambiguous, nano-spectroscopic analysis indicates the $\mathrm{Nb}$ bottom electrode to 
be partially oxidized (Figure 2) and to be most likely a $\mathrm{Nb}$ (II) oxide which is known to be almost metallic.[31] Comparative measurements have been done, using an $\mathrm{Al}_{2} \mathrm{O}_{3}-\mathrm{Al} / \mathrm{Nb}$ and a $\mathrm{Nb}_{\mathrm{x}} \mathrm{O}_{\mathrm{y}} / \mathrm{Al}_{2} \mathrm{O}_{3}-\mathrm{Al} / \mathrm{Nb}$ stack. The additional $\mathrm{Nb}_{\mathrm{x}} \mathrm{O}_{\mathrm{y}}$ turned out not to influence the stack resistance substantially. However, although this is a strong indicator, it does not prove a possible oxidation of the $\mathrm{Nb}$ electrode does not influence the overall device behavior of the DBM device. To further investigate the influence of a partially oxidized $\mathrm{Nb}$ electrode, a lumped element circuit model has been set up and solved with the simulation tool SPICE.

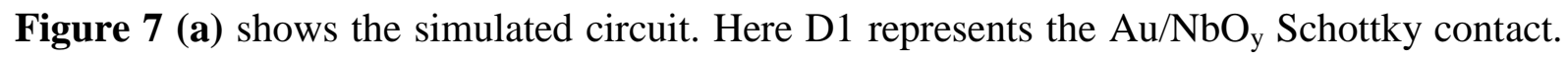
The underlying equation, describing the current through the Schottky contact, following the thermionic emission theory, is given by:

$$
\begin{gathered}
I_{S}=I_{R} \cdot \exp \left(\frac{e V}{n k_{B} T}-1\right) \\
I_{R}(V>0)=\mathrm{A} \cdot \mathrm{AT}^{2} \cdot \exp \left(\frac{-\Phi_{b}}{k_{B} T}\right) \\
I_{R}(V<0)=-\mathrm{A} \cdot \mathrm{AT}^{2} \cdot \exp \left(\frac{-\Phi_{b}}{k_{B} T}\right) \exp \left(\frac{\alpha_{r} \sqrt{|V|}}{k_{B} T}\right)
\end{gathered}
$$

where $\Phi_{b}$ is the Schottky barrier height $(0.83 \mathrm{eV}), e$ is the elementary charge, $A$ is the junction area, $k_{B}$ and $T$ are the Boltzmann constant and the temperature, $A *$ is the effective Richardson constant $\left(1.20173 * 10^{6} \mathrm{~A} / \mathrm{m}^{2} \mathrm{~K}^{2}\right), n$ is the ideality factor (3.6), $\alpha_{r}$ denotes a device dependent parameter (0.15) and $V$ is the voltage drop over the Schottky contact. The parameters of the circuit have been chosen so that the device is in the low resistance state as presented in [18]. The resistance $\mathrm{R}_{\mathrm{t}}$ (t stands for the top $\mathrm{Nb}$ oxide layer) represents the $\mathrm{NbO}_{\mathrm{y}}$ layer. The resistance is calculated by:

$$
R_{t}=\frac{\rho_{t} l_{t}}{A}
$$


Where $l_{\mathrm{t}}$ is the thickness and $\rho_{\mathrm{t}}$ is the specific resistance $\left(2.5 \cdot 10^{3} \Omega \mathrm{m}\right)$ of the $\mathrm{NbO}_{\mathrm{y}}$ layer. The varistor VDR represents the $\mathrm{AlO}_{\mathrm{x}}$ tunnel barrier. The current through the tunnel barrier is given by the current formula of Simmons:

$$
I_{T}=\frac{A e}{2 \pi h d} \cdot\left(x_{1} \cdot \exp \left[-\frac{4 \pi d \sqrt{2 m}}{h} \sqrt{x_{1}}\right]-x_{2} \cdot e \exp \left[-\frac{4 \pi d \sqrt{2 m}}{h} \sqrt{x_{2}}\right]\right)
$$

with $x_{1}=e \Phi_{0}-e V / 2$ and $x_{2}=e \Phi_{0}+e V / 2 . \Phi_{0}$ is the barrier height of the tunnel barrier (3.1 eV), $V$ is the voltage drop over the tunnel barrier, $m$ is the electron mass, $h$ is Planck's constant and $d$ is the thickness of the tunnel barrier. Up to now, this model is very similar to the electrical model presented in [16]. Within this work, this model has been extended by a second resistance $\mathrm{R}_{\mathrm{b}}$ (b for bottom), representing the partially oxidized $\mathrm{Nb}$ electrode. This resistance is calculated by:

$$
R_{b}=\frac{\rho_{b} l_{b}}{A}
$$

Here $l_{\mathrm{b}}$ is the thickness of the oxidized part of the $\mathrm{Nb}$ electrode (measured to be about $6.5 \mathrm{~nm}$ ) and $\rho_{\mathrm{b}}$ is the specific resistance of the $\mathrm{NbO}_{\mathrm{z}}$ layer.

The voltage drop over the $\mathrm{NbO}_{\mathrm{y}}$ part (represented by $\mathrm{R}_{\mathrm{t}}$ ) gives deeper insights into the behaviour of the global device. At first, the applied voltage drop leads to an electric field within the $\mathrm{NbO}_{\mathrm{y}}$ layer. This electric field itself leads to a motion of oxygen vacancies and thus to a resistance change of the device as presented in [18]. This means, the higher the voltage drop over the $\mathrm{NbO}_{\mathrm{y}}$ part, the higher the resulting electric field and the higher the resistance change. Hence, the voltage drop provides information about the resistive switching. Furthermore, due to the series like circuit, the current through the device is proportional to the voltage drop over the $\mathrm{NbO}_{\text {y }}$ part.Hence, the voltage drop over the $\mathrm{NbO}_{\text {y }}$ part also provides information about the device current. The resistance $\mathrm{R}_{\mathrm{b}}$ influences the voltage drop over the resistance $\mathrm{R}_{t}$ and therefore over the $\mathrm{NbO}_{y}$ part. Therefore, at first the voltage drop over $\mathrm{R}_{t}$ is calculated without $R_{b}$ for two different voltages applied to the overall device $(2 \mathrm{~V}$ and $3 \mathrm{~V})$. 
Afterwards, the voltage drop over $R_{t}$ is calculated including $R_{b}$ to the circuit also for $2 \mathrm{~V}$ and $3 \mathrm{~V}$ respectively. The change of the voltage drop over $\mathrm{R}_{t}$ is a measure for the influence of the $\mathrm{NbO}_{z}$ part. Since the $\mathrm{NbO}_{z}$ layer was measured to be $\mathrm{Nb}(\mathrm{II})$ oxide, the specific resistance $\rho_{\mathrm{b}}$ is expected to be on the order of $10^{-6} \Omega \mathrm{m}$. [33] However, the exact specific resistance is not known. Therefore, the voltage drop over $R_{t}$ is calculated for different values of $R_{b}$, resulting from different $\rho_{\mathrm{b}}$ ranging from $10^{-6} \Omega \mathrm{m}$ up to $10^{4} \Omega \mathrm{m}$.

Figure 7 (c) shows the relative voltage drop $\mathrm{V} / \mathrm{V}_{0}$ over the $\mathrm{NbO}_{\mathrm{y}}$ layer for different specific resistances $\rho_{\mathrm{b}}$ of $\mathrm{R}_{\mathrm{b}}$. As a result, up to a specific resistance of $10^{2} \Omega \mathrm{m}$, which is already the specific resistance of $\mathrm{Nb}(\mathrm{IV})$ oxide, there is nearly no change in the voltage drop over the NbOy layer. For higher specific resistances the relative voltage drop changes strongly, indicating a large influence of $\mathrm{R}_{\mathrm{b}}$ on the switching mechanism. Smaller applied voltages lead to a lower change in the relative voltage. This is shown for an example voltage of $2 \mathrm{~V}$. As long as the change in relative voltage drop is small, the voltage drop over the $\mathrm{NbOz}$ layer is negligible. This results in a small electric field. Under this condition, the motion of defects within this layer can be neglected, due to the negligible electric field. The simulation results underline that the partially oxidized $\mathrm{Nb}$ electrode influences the overall behaviour of the device only marginally.

\section{DISCUSSION}

As apparent from Figure 2 the interfaces around the top $\mathrm{NbO}_{\mathrm{y}}$ layer are extremely smooth with virtually no detectable surface roughness. This is extremely important for the proposed switching mechanism, as it ascertains homogenous, undistorted electrical fields, in turn allowing for homogenous memristive switching. Furthermore, the investigations underline the high oxidation state of the $\mathrm{NbO}_{\mathrm{y}}$ layer. Being at least $\mathrm{Nb}(\mathrm{IV})$ oxide, this ensures a functioning Schottky barrier between the Au top electrode and $\mathrm{NbO}_{\mathrm{y}}$ especially in combination with the layer thickness, closely corresponding to the targeted values. 
As for the oxidized bottom electrode, sub-oxides of $\mathrm{Nb}$ are known to be either n-type semiconductors or even metallic conductors. Thus, the existence of pronounced $\mathrm{Nb} \mathrm{BE}$ oxidation does not hamper the memristive mechanism proposed by Hansen et al, as also underlined by the electrical simulations and anodization spectroscopy. The reason for oxidation of the BE cannot be resolved with certainty. Given that $\mathrm{Al}$ is known to exhibit selflimiting oxidation [37] at room temperature and standard atmosphere of around only $1 \mathrm{~nm}$, it seems plausible that another external driving force is required to oxidize the Al completely. Observation of this self-limited oxidation in the Josephson junction (cf. supplement) suggests that through-oxidation of the $\mathrm{Al}$ and the oxidation of the $\mathrm{BE}$ is linked to the additional deposition steps, namely the reactive deposition of $\mathrm{NbO}_{\mathrm{y}}$. Here, the Ar/O plasma ignited for reactive deposition can serve as a source for oxidation of the underlying layers, the driving force being a combination of concentration gradient from high (plasma) to low $\left(\mathrm{AlO}_{\mathrm{x}}\right.$ and at the time of deposition the metallic $\mathrm{Nb} \mathrm{BE}$ ) and the kinetic energy and charge of the oxygen ions. It should be noted that despite this difference in targeted and actual layer sequence the device still exhibits memristive properties as described by Hansen et al, underlining the superior robustness to deviations of the DBM approach. Anodization spectroscopy data further substantiate this by proving the metallic character of the bottom electrode and underlining that reactive sputtering above a metallic $\mathrm{Nb}$ electrode has no significant influence on electrical behaviour.

The finding of $\mathrm{O}$ vacancies in the $\mathrm{AlO}_{\mathrm{x}}$ tunnel barrier suggests that this thought to be passive layer might change its resistivity during bias application, after all. However, the number of vacancies is expected to be low enough to not have an influence on tunnelling behaviour. In particular, the amorphous nature of the tunnel barrier implies that these defects have little to no effect as discussed by Pesenson et al. [38] The lowest calculated defect concentration of $1.7 \%$ results in a theoretical average inter-defect distance of $29.5 \AA$. Considering the tunnelling thickness of $1 \mathrm{~nm}$, this inter-defect distance is expected to be large enough to not 16 
have an influence. Potential existence of $\mathrm{O}$ vacancies in either of the $\mathrm{Nb}$ oxide layers cannot be measured in the same way. Detection of oxygen vacancies in $\mathrm{AlO}_{\mathrm{x}}$ relies on their effect of inter-band states. Only if $\mathrm{O}$ vacancies in $\mathrm{Nb}$ oxide had the same effect would they be detectable in a similar fashion.

The discrepancy of potentially finding metallic Al during anodization spectroscopy and its absence in STEM investigations can be caused by different factors. Anodization spectroscopy was conducted with large scale samples; hence the result could imply that TEM samples were taken from randomly oxidized regions. However, during the course of investigations around ten lamellae were extracted from different DBM devices and none of them showed metallic $\mathrm{Al}$, indeed all samples exhibit congruent results. On the other hand, anodization spectroscopy relies on the change of resistivity characteristic for different materials. Yet, no complete theoretical description or the possibility of simulation for these spectra exist, potentially hampering correct interpretation. The surface roughness between the $\mathrm{Al}$ und $\mathrm{Nb}$ oxides might simply render the thicker $\mathrm{AlO}_{\mathrm{x}}$ regions undetectable for anodization spectroscopy and the effect of a metallically-conducting $\mathrm{Nb}$ suboxide is also unknown.

\section{CONCLUSION}

Intensive spectroscopic investigations on memristive $\mathrm{AlO}_{\mathrm{x}} / \mathrm{NbO}_{\mathrm{y}}$ devices and their Josephson junction base were conducted.

1. Slight oxidation of the BE was found. As the layer sequence of the Josephson junction base has been verified beforehand, oxidation possibly took place in the subsequent deposition of $\mathrm{NbO}_{\mathrm{y}}$ on top of the junction's $\mathrm{AlO}_{\mathrm{x}}$ tunnel barrier. Simulations in accordance with EELS and anodization spectroscopy underline the low resistance of this layer. 
2. Oxidation due to TEM preparation artifacts has been excluded by a comprehensive approach including low energy Argon ion thinning by PIPS, standard FIB lamella thinning, and backside FIB lamella thinning.

3. An unexpected content of oxygen vacancies in the $\mathrm{AlO}_{\mathrm{x}}$ was found its effect on memristive behaviour is unknown. It was not possible to test for oxygen vacancies in $\mathrm{Nb}$ oxide in the same manner.

4. Nano-spectroscopic investigations showed that surface roughness limits the thickness of the tunnel barrier to around $1 \mathrm{~nm}$ and the Schottky barrier forming $\mathrm{NbO}_{\mathrm{y}}$ to around $2 \mathrm{~nm}$, thus closely corresponding to the targeted layer design. Importantly, surface roughness around the Schottky barrier forming layers is virtually zero, thus ensuring a homogenous electrical field distribution and in turn homogenous memristive switching.

Overall, these results demonstrate the high robustness of the DBM regarding deviations from the targeted design and layer sequence. However, they also open up the path towards potential improvements.

- Deposition of a thicker Al layer could inhibit the oxidation of the bottom electrode.

- An additional adhesion layer might reduce the surface roughness visible between the (oxidized) $\mathrm{Nb}$ bottom electrode and the Al layer. Alternatively, deposition of Al oxide by atomic layer deposition has been shown to produce atomically smooths layers.

- The latter could also resolve the presence of oxygen vacancies in the tunnel barrier. 


\section{ACKNOWLEDGEMENTS}

The authors gratefully acknowledge funding by the DFG through the research unit FOR2093 and by the European Union within the 7th Framework Program (FP7/2007-2013) under Grant Agreement no. 312483 (ESTEEM2). JS and LK would like to thank Mrs. Christin Szillus and Mrs. Martina Dienstleder for sample preparation by FIB and NanoMill and Prof. Ferdinand Hofer as well as Prof. Dagmar Gerthsen for supporting the investigations. The authors acknowledge the support of the Karlsruhe Nano Micro Facility (KNMF) for Electron Microscopy and Spectroscopy facilities.

\section{REFERENCES}

[1] D.B. Strukov, G.S. Snider, D.R. Stewart, R.S. Williams, The missing memristor found, Nature. 453 (2008) 80-83. doi:10.1038/nature06932.

[2] L. Chua, Memristor-The missing circuit element, IEEE Trans. Circuit Theory. 18 (1971) 507-519. doi:10.1109/TCT.1971.1083337.

[3] D. Ielmini, R. Waser, Resistive Switching: From Fundamentals of Nanoionic Redox Processes to Memristive Device Applications, John Wiley \& Sons, 2015.

[4] R. Tetzlaff, Memristors and Memristive Systems, 2014. http://www.springer.com/us/book/9781461490678 (accessed December 20, 2016).

[5] A. Adamatzky, L. Chua, Memristor Networks, Springer International Publishing, Bristol, 2014.

[6] Q. Xia, Nanoscale resistive switches: devices, fabrication and integration, Appl. Phys. A. 102 (2011) 955-965. doi:10.1007/s00339-011-6288-1.

[7] P. Calka, E. Martinez, V. Delaye, D. Lafond, G. Audoit, D. Mariolle, N. Chevalier, H. Grampeix, C. Cagli, V. Jousseaume, C. Guedj, Chemical and structural properties of conducting nanofilaments in TiN/HfO 2 -based resistive switching structures, Nanotechnology. 24 (2013) 085706. doi:10.1088/0957-4484/24/8/085706.

[8] Y. Yang, P. Gao, S. Gaba, T. Chang, X. Pan, W. Lu, Observation of conducting filament growth in nanoscale resistive memories, Nat. Commun. 3 (2012) 732. doi:10.1038/ncomms1737.

[9] X. Tian, L. Wang, J. Wei, S. Yang, W. Wang, Z. Xu, X. Bai, Filament growth dynamics in solid electrolyte-based resistive memories revealed by in situ TEM, Nano Res. 7 (2014) 1065-1072. doi:10.1007/s12274-014-0469-0.

[10] Q. Liu, S. Long, H. Lv, W. Wang, J. Niu, Z. Huo, J. Chen, M. Liu, Controllable Growth of Nanoscale Conductive Filaments in Solid-Electrolyte-Based ReRAM by Using a Metal Nanocrystal Covered Bottom Electrode, ACS Nano. 4 (2010) 6162-6168. doi:10.1021/nn1017582.

[11] T. Ninomiya, Z. Wei, S. Muraoka, R. Yasuhara, K. Katayama, T. Takagi, Conductive Filament Scaling of Bipolar ReRAM for Improving Data Retention Under Low Operation Current, IEEE Trans. Electron Devices. 60 (2013) 1384-1389. doi:10.1109/TED.2013.2248157. 
[12] Y. Aoki, C. Wiemann, V. Feyer, H.-S. Kim, C.M. Schneider, H. Ill-Yoo, M. Martin, Bulk mixed ion electron conduction in amorphous gallium oxide causes memristive behaviour, Nat. Commun. 5 (2014) 3473. doi:10.1038/ncomms4473.

[13] P. Zalden, M.J. Shu, F. Chen, X. Wu, Y. Zhu, H. Wen, S. Johnston, Z.-X. Shen, P. Landreman, M. Brongersma, S.W. Fong, H.-S.P. Wong, M.-J. Sher, P. Jost, M. Kaes, M. Salinga, A. von Hoegen, M. Wuttig, A.M. Lindenberg, Picosecond Electric-FieldInduced Threshold Switching in Phase-Change Materials, Phys. Rev. Lett. 117 (2016) 067601. doi:10.1103/PhysRevLett.117.067601.

[14] D.-H. Kwon, K.M. Kim, J.H. Jang, J.M. Jeon, M.H. Lee, G.H. Kim, X.-S. Li, G.-S. Park, B. Lee, S. Han, M. Kim, C.S. Hwang, Atomic structure of conducting nanofilaments in $\mathrm{TiO} 2$ resistive switching memory, Nat. Nanotechnol. 5 (2010) 148-153. doi:10.1038/nnano.2009.456.

[15] A. Chanthbouala, V. Garcia, R.O. Cherifi, K. Bouzehouane, S. Fusil, X. Moya, S. Xavier, H. Yamada, C. Deranlot, N.D. Mathur, M. Bibes, A. Barthélémy, J. Grollier, A ferroelectric memristor, Nat. Mater. 11 (2012) 860-864. doi:10.1038/nmat3415.

[16] M. Hansen, M. Ziegler, L. Kolberg, R. Soni, S. Dirkmann, T. Mussenbrock, H. Kohlstedt, A double barrier memristive device, Sci. Rep. 5 (2015) 13753.

[17] H.Y. Jeong, J.Y. Lee, S.-Y. Choi, J.W. Kim, Microscopic origin of bipolar resistive switching of nanoscale titanium oxide thin films, Appl. Phys. Lett. 95 (2009) 162108. doi:10.1063/1.3251784.

[18] S. Dirkmann, M. Hansen, M. Ziegler, H. Kohlstedt, T. Mussenbrock, The role of ion transport phenomena in memristive double barrier devices, Sci. Rep. 6 (2016) 35686. doi:10.1038/srep35686.

[19] F. Peña, T. Ostasevicius, V.T. Fauske, P. Burdet, P. Jokubauskas, M. Sarahan, D. Johnstone, M. Nord, J. Taillon, J. Caron, K.E. MacArthur, A. Eljarrat, S. Mazzucco, T. Furnival, E. Prestat, M. Walls, G. Donval, B. Martineau, L.F. Zagonel, A. Garmannslund, T. Aarholt, C. Gohlke, iygr, hyperspy: HyperSpy 1.1, (2016). http://dx.doi.org/10.5281/zenodo.59293.

[20] M. Bosman, M. Watanabe, D.T.L. Alexander, V.J. Keast, Mapping chemical and bonding information using multivariate analysis of electron energy-loss spectrum images, Ultramicroscopy. 106 (2006) 1024-1032. doi:10.1016/j.ultramic.2006.04.016.

[21] T. Imamura, S. Hasuo, Fabrication of High Quality $\mathrm{Nb} / \mathrm{AlOx}-\mathrm{Al} / \mathrm{Nb}$ Josephson Junctions: II-Deposition of Thin Al Layers on Nb Films, IEEE Trans. Appl. Supercond. 2 (1992) 84-94. doi:10.1109/77.139224.

[22] R.E. Joynson, C.A. Neugebauer, J.R. Rairden, Thin Films of Niobium for Cryotron Ground Planes, J. Vac. Sci. Technol. 4 (1967) 171-178. doi:10.1116/1.1492542.

[23] H.A. Huggins, M. Gurvitch, Preparation and characteristics of Nb/Al-oxide-Nb tunnel junctions, J. Appl. Phys. 57 (1985) 2103-2109. doi:10.1063/1.334403.

[24] N. Jiang, J.C.H. Spence, Interpretation of Oxygen K pre-edge peak in complex oxides, Ultramicroscopy. 106 (2006) 215-219. doi:10.1016/j.ultramic.2005.07.004.

[25] M. Klimenkov, A. Möslang, R. Lindau, EELS analysis of complex precipitates in PM 2000 steel, Eur. Phys. J. Appl. Phys. 42 (2008) 293-303. doi:10.1051/epjap:2008084.

[26] J. Bruley, R. Brydson, H. Müllejans, J. Mayer, G. Gutekunst, W. Mader, D. Knauss, M. Rühle, Investigations of the chemistry and bonding at niobiumsapphire interfaces, J. Mater. Res. 9 (1994) 2574-2583. doi:10.1557/JMR.1994.2574.

[27] S. Nigo, M. Kubota, Y. Harada, T. Hirayama, S. Kato, H. Kitazawa, G. Kido, Conduction band caused by oxygen vacancies in aluminum oxide for resistance random access memory, J. Appl. Phys. 112 (2012) 033711. doi:10.1063/1.4745048.

[28] J.A. Tossell, The electronic structures of $\mathrm{Mg}, \mathrm{Al}$ and $\mathrm{Si}$ in octahedral coordination with oxygen from SCF X $\alpha$ MO calculations, J. Phys. Chem. Solids. 36 (1975) 1273-1280. doi:10.1016/0022-3697(75)90203-6. 
[29] D. Liu, J. Robertson, Oxygen vacancy levels and interfaces of A12O3, Microelectron. Eng. 86 (2009) 1668-1671. doi:10.1016/j.mee.2009.03.011.

[30] H. Momida, S. Nigo, G. Kido, T. Ohno, Effect of vacancy-type oxygen deficiency on electronic structure in amorphous alumina, Appl. Phys. Lett. 98 (2011) 042102. doi:10.1063/1.3548549.

[31] V.A. Pustovarov, V.S. Aliev, T.V. Perevalov, V.A. Gritsenko, A.P. Eliseev, Electronic Structure of an Oxygen Vacancy in $\mathrm{Al} 2 \mathrm{O} 3$ from the Results of Ab Initio QuantumChemical Calculations and Photoluminescence Experiments, J. Exp. Theor. Phys. 111 (2010) 989-995.

[32] K.H. Johnson, S.V. Pepper, Molecular-orbital model for metal-sapphire interfacial strength, J. Appl. Phys. 53 (1982) 6634-6637. doi:10.1063/1.330095.

[33] D. Bach, EELS investigations of stoichiometric niobium oxides and niobium-based capacitors, Universität Karlsruhe, 2009.

[34] R. Brydson, Multiple scattering theory applied to ELNES of interfaces, J. Phys. Appl. Phys. 29 (1996) 1699. doi:10.1088/0022-3727/29/7/004.

[35] T. Imamura, S. Hasuo, Characterization of $\mathrm{Nb} / \mathrm{AlOx}-\mathrm{Al} / \mathrm{Nb}$ Josephson junctions by anodization profiles, J. Appl. Phys. 66 (1989) 2173-2180. doi:doi:10.1063/1.344314.

[36] J.M. Rowell, M. Gurvitch, J. Geerk, Modification of tunneling barriers on Nb by a few monolayers of Al, Phys. Rev. B. 24 (1981) 2278-2281. doi:10.1103/PhysRevB.24.2278.

[37] N. Cabrera, N.F. Mott, Theory of the oxidation of metals, Rep. Prog. Phys. 12 (1949) 163. doi:10.1088/0034-4885/12/1/308.

[38] L. Pesenson, R.P. Robertazzi, R.A. Buhrman, S.R. Cypher, B.D. Hunt, Interacting and self-organized two-level states in tunnel barriers, Phys. Rev. Lett. 67 (1991) 2866-2869. doi:10.1103/PhysRevLett.67.2866. 

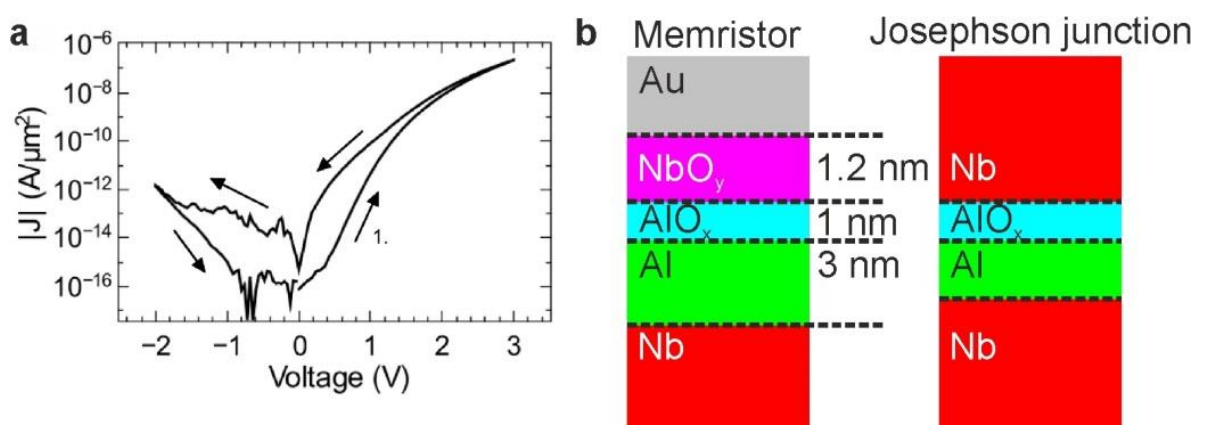

Figure 1: The DBM device as presented by Hansen et al (2015). (a) Measured I-V curve with distinct low- and high-resistance states. (b) Layer scheme and approximate thicknesses expected from deposition parameters for the memristor. (c) Design-wise similar Josephson junction which was analysed in this study because of its comparability to the DBM.

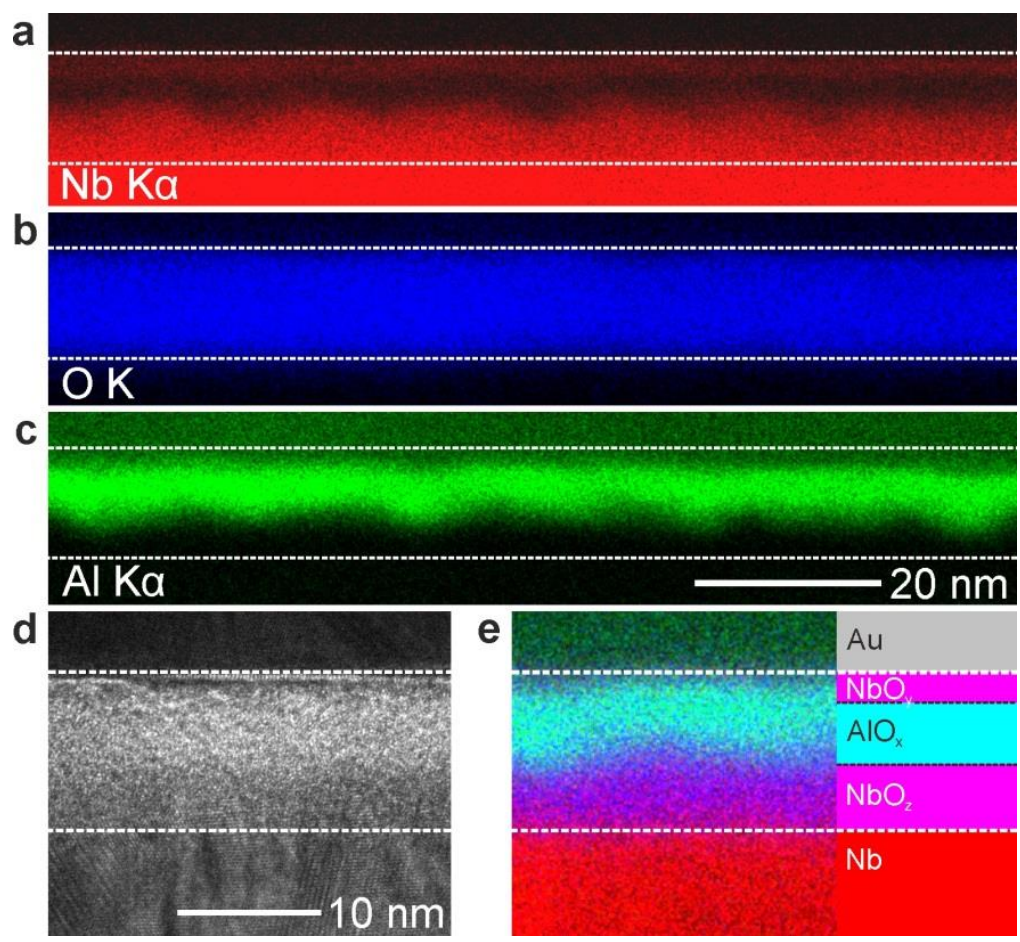

Figure 2: Structural and chemical analysis of the DBM. (a) to (c) show STEM-EDX maps of $\mathrm{Nb}, \mathrm{O}$ and $\mathrm{Al}$ respectively taken from a backside thinned sample. To enhance visibility of the $\mathrm{NbO}_{\mathrm{y}}$ layer, intensity, contrast and brightness in (a) have been modified. Dashed white lines indicate the extent of the oxide layers. (d) HRTEM micrograph showing the crystalline nature of the $\mathrm{Au}$ and $\mathrm{Nb}$ electrodes and amorphous character of the oxide layers between. (e) Compound map created from maps (a) to (c) proving that the amorphous region in (d) 
corresponds to the $\mathrm{NbO}_{\mathrm{y}} / \mathrm{AlO}_{\mathrm{x}} / \mathrm{NbO}_{\mathrm{z}}$ layers next to a sketch (not to scale) of the observed layer sequence as opposed to the sequence shown in Figure 1.
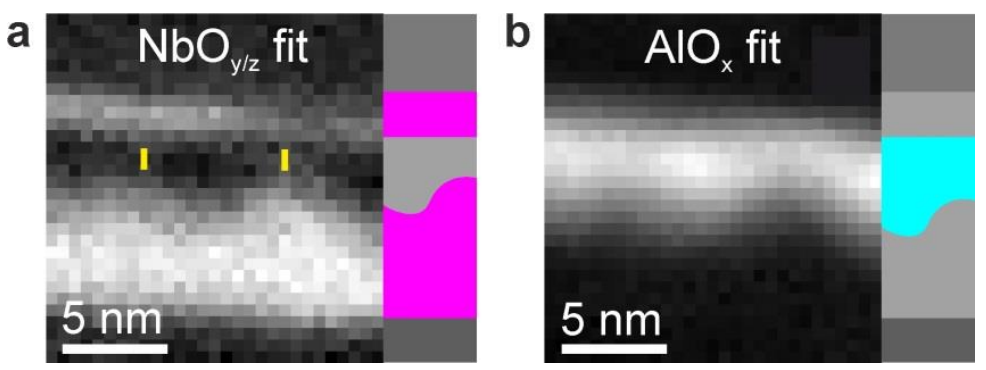

Figure 3: Goodness of fit map of the $\mathrm{O}-\mathrm{K}$ edge with an $\mathrm{O}-\mathrm{K}$ edge reference of (a) $\mathrm{NbO}_{\mathrm{y} / \mathrm{z}}$ and (b) $\mathrm{AlO}_{\mathrm{x}}$. The references with which the fit was performed were extracted from data of the same set and references from several samples are displayed in Figure S1. While the EDX maps in Figure 2 and the AlOx fit in (b) suggest that the $\mathrm{AlOx}$ is around $5 \mathrm{~nm}$ thick, the $\mathrm{Nb}$ oxide fit in (a) proves that surface roughness effects limits the tunnel barrier thickness to around $1 \mathrm{~nm}$ at the thinnest spots. The yellow lines are $1 \mathrm{~nm}$ and indicate the thinnest locations in the tunnel barrier. For a detailed description of data handling cf. section II.

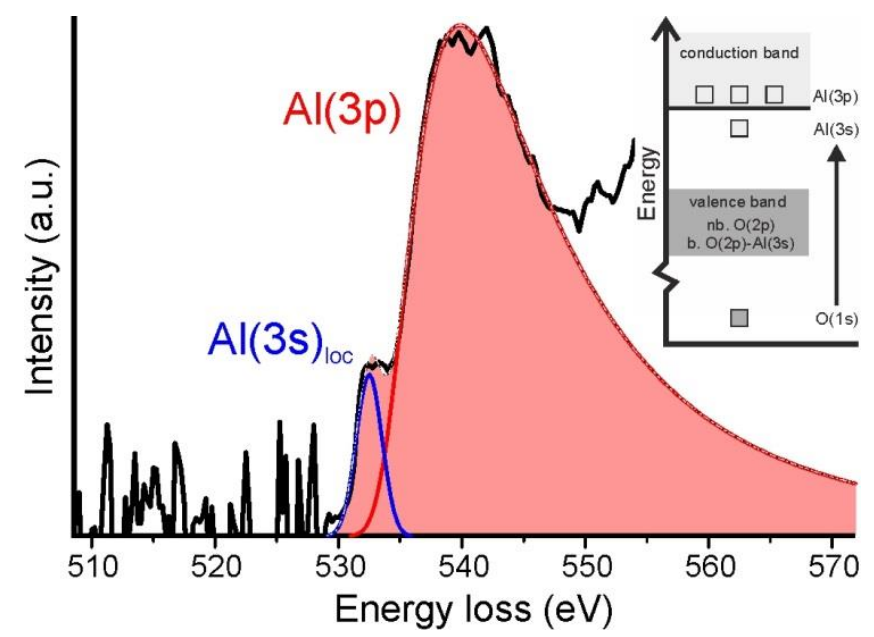

Figure 4: Close up of the $\mathrm{O}-\mathrm{K}$ edge of the $\mathrm{AlO}_{\mathrm{x}}$ region including the fit line. Spectrum has undergone background subtraction prior to fitting. A Gaussian peak (blue) was chosen for the satellite feature and a Pearson IV function (red) was fit to the main edge of oxygen, the combined fit is indicated by the red area. The ratio of cross sections is $4.3 \%$. Inset shows a reduced diagram of the excitation process from $\mathrm{O}(1 \mathrm{~s})$ to the $\mathrm{Al}$ states above the Fermi energy. 


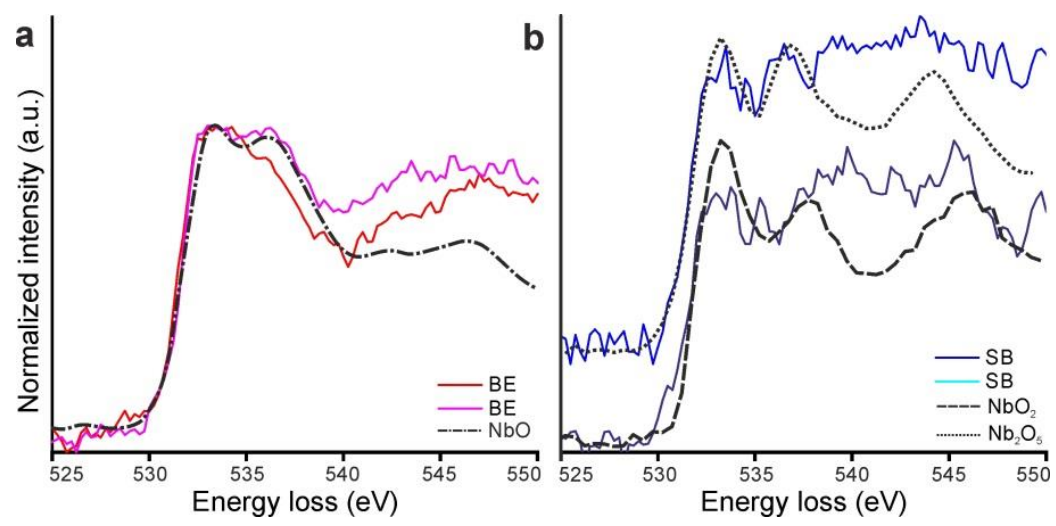

Figure 5: EEL spectra containing the $\mathrm{O} \mathrm{K}$ edge of different samples, extracted from $\mathrm{Nb}$ oxide containing regions. a) The shape of the $\mathrm{NbO}_{\mathrm{y}}$ bottom electrode ELNES matches the fingerprints of $\mathrm{Nb}(\mathrm{II})$ oxide. b) The Schottky barrier oxide is most likely a mixture of oxidation states $\mathrm{Nb}(\mathrm{IV})$ and $\mathrm{Nb}(\mathrm{V})$. The net intensity deviation a higher energy losses is likely due to incorrect background subtraction and plural scattering effects.

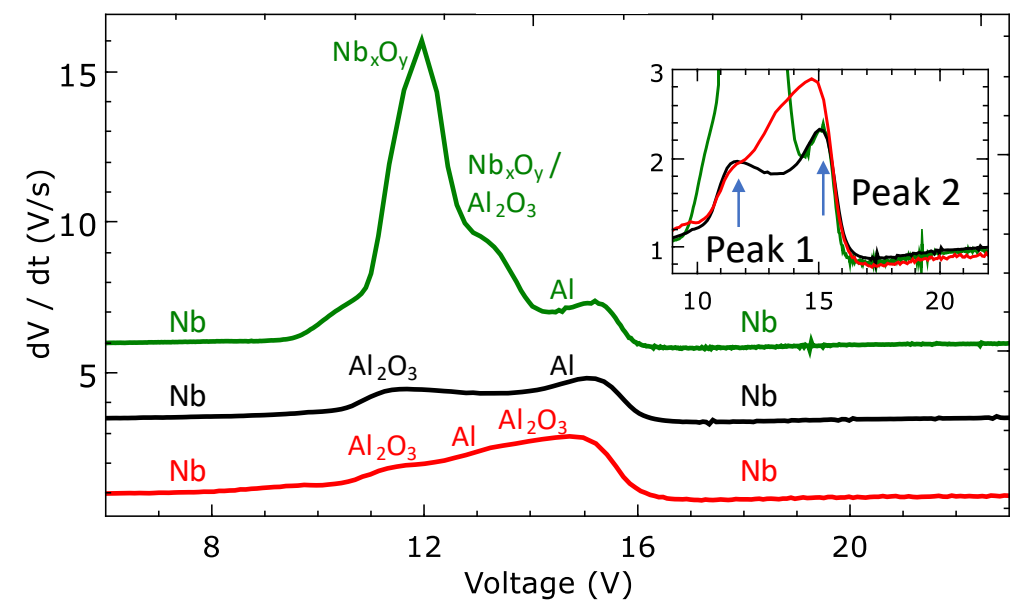

Figure 6: Anodization spectra of a Josephson junction (black curve), the DBM (green curve) and a sample to show the influence on the anodization spectroscopy of a partially oxidized aluminum layer (red curve). All curves were normalized to an anodization rate of $1 \mathrm{~V} / \mathrm{s}$ for niobium. The spectra were vertically shifted for a better illustration. The inset shows all curves on top of each other, to visualize the different anodization rates around peak 2. 


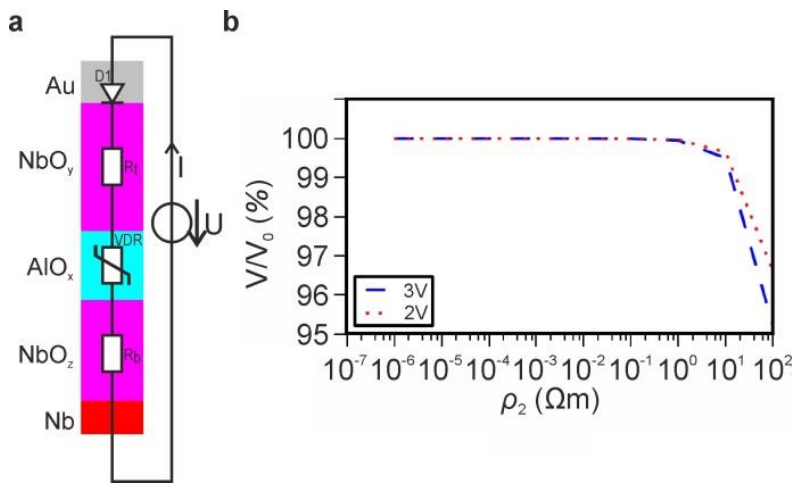

Figure 7: Input and results for kinetic Monte Carlo simulations. (a) Lumped element model of the DBM device. D1 represents the Schottky contact, $\mathrm{R}_{t}$ the $\mathrm{NbO}_{\mathrm{y}}$ part, VDR the $\mathrm{Al}_{2} \mathrm{O}_{3}$ tunnel barrier and $\mathrm{R}_{\mathrm{b}}$ the oxidized $\mathrm{Nb}$ electrode. (b) Voltage drop $\mathrm{V}$ over the $\mathrm{NbO}_{\mathrm{y}}$ part including $\mathrm{R}_{\mathrm{b}}$ in terms of the voltage drop $\mathrm{V}_{0}$ over the $\mathrm{NbO}_{\mathrm{y}}$ part without $\mathrm{R}_{\mathrm{b}}$ for different specific resistances $\rho_{b}$ of $R_{b}$. 